\title{
TROPICAL CURVES, THEIR JACOBIANS AND THETA FUNCTIONS
}

\author{
GRIGORY MIKHALKIN AND ILIA ZHARKOV
}

\begin{abstract}
We study Jacobian varieties for tropical curves. These are real tori equipped with integral affine structure and symmetric bilinear form. We define tropical counterpart of the theta function and establish tropical versions of the Abel-Jacobi, Riemann-Roch and Riemann theta divisor theorems.
\end{abstract}

\section{INTRODUCTION}

In this paper we study algebraic curves defined over the so-called tropical semifield

$$
\mathbb{T}=\mathbb{R} \cup\{-\infty\}
$$

equipped with the following tropical arithmetic operations for $x, y \in \mathbb{T}$.

$$
\text { " } x+y "=\max \{x, y\}, " x y "=x+y .
$$

Here we use the quotation marks to distinguish between the classical and tropical operations. It is easy to check that each of these operations is commutative and associative and that together they satisfy to the distribution law.

These arithmetic operation do not turn $\mathbb{T}$ into a field. The idempotency of addition, " $x+x "=x$ makes subtraction impossible. However it does admit a division: $" \frac{x}{y} "=x-y$ once $y \neq-\infty$. Furthermore, $\mathbb{T}$ has the additive zero " $0_{\mathbb{T}} "=-\infty$ and the multiplicative unit " $1_{\mathbb{T}}$ " $=0$. We say that $\mathbb{T}$ is a semifield as we have all the operations except for subtraction.

It is possible to define algebraic varieties over $\mathbb{T}$ in a similar way as one defines algebraic varieties over fields, e.g. $\mathbb{C}$ or $\mathbb{R}$, see $[\mathrm{Mik}$. In this paper, we are concerned only with tropical curves which we define in the next section.

Tropical arithmetic operations are related to classical ones via the so-called dequantization. Namely let us define a family of addition operations on $\mathbb{R} \cup\{-\infty\}$ by

$$
x \oplus_{t} y=\log _{t}\left(t^{x}+t^{y}\right),
$$

where $t$ is the parameter ranging from $e$ to $+\infty$. For any finite value of $t$ this operation is (by its definition) induced from $\mathbb{R}_{\geq 0}=\{z \in \mathbb{R} \mid z>0\}$. Clearly, $\log _{t}\left(t^{x} t^{y}\right)=x+y$ and thus $\mathbb{R} \cup\{-\infty\}$ equipped with $\oplus_{t}$ for addition and + for

The first author is supported in part by NSERC and the second author is supported in part by NSF grant DMS-0405939. 
multiplication is isomorphic (as a semifield) to $\mathbb{R}_{\geq 0}$ with the usual arithmetical operations. However, we have

$$
\lim _{t \rightarrow+\infty} \log _{t}\left(t^{x}+t^{y}\right)=\max \{x, y\}
$$

which exhibits $\mathbb{T}$ as the limit semifield.

This degeneration of addition in $\mathbb{R}_{\geq 0}$ underlies the following collapse of complex algebraic varieties (see [KS01] and [GW00] for the case of Calabi-Yau varieties) in $\left.\left(\mathbb{C}^{*}\right)^{n}\right)$. Let

$$
K=\left\{a(t)=\sum_{j \in I} \alpha_{j} t^{j} \mid \alpha_{j} \in \mathbb{C}\right\}
$$

here $I \subset \mathbb{R}$ is countable and well-ordered and $a(t)$ converges for $t \in\left[0, t_{0}\right], t_{0}>0$. Such $K$ is an example of the so-called non-Archimedean field: there exist a valuation, i.e. a function

$$
\text { val }: K=K \rightarrow \mathbb{T}
$$

such that $\operatorname{val}^{-1}(-\infty)=0_{K}$ and for any $z, w \in K$ we have $\operatorname{val}(z+w) \leq{ }^{\prime} \operatorname{val}(z)+$ $\operatorname{val}(w) "=\max \{\operatorname{val}(z), \operatorname{val}(w)\}$ and $\operatorname{val}(z w)=" \operatorname{val}(z) \operatorname{val}(w) "=\operatorname{val}(z)+\operatorname{val}(w)$. We have the valuation map

$$
\text { Val : }\left(K^{*}\right)^{n} \rightarrow \mathbb{R}^{n},
$$

where $K^{*}=K \backslash\{0\}$, defined by $\operatorname{Val}\left(z_{1}, \ldots, z_{n}\right)=\left(\operatorname{val}\left(z_{1}\right), \ldots, \operatorname{val}\left(z_{n}\right)\right)$.

Let $V \subset\left(K^{*}\right)^{n}$ be an algebraic variety over $K$. Following Kapranov Kap00 one may associate to $V$ its non-Archimedean amoeba, i.e. the image $\mathcal{A}=\operatorname{Val}(V) \subset \mathbb{R}^{n}$. For small values of $t>0$ the variety $V$ determines a complex algebraic variety $V_{t} \subset\left(\mathbb{C}^{*}\right)^{n}$ by plugging $t$ to all converging series in $t$ that form the coordinates of $V \subset\left(K^{*}\right)^{n}$. According to Gelfand, Kapranov and Zelevinski GKZ94] one may also associate to $V_{t}$ its amoeba by taking the image $\mathcal{A}_{t}=\log _{t}\left(V_{t}\right) \subset \mathbb{R}^{n}$, where

$$
\log _{t}:\left(\mathbb{C}^{*}\right)^{n} \rightarrow \mathbb{R}^{n}
$$

is defined by $\log _{t}\left(z_{1}, \ldots, z_{n}\right)=\left(\log _{t}\left(z_{1}\right), \ldots, \log _{t}\left(z_{n}\right)\right)$. Note that the base $t$ of the logarithm coincides with the parameter of deformation of $V_{t}$. We have the following diagram

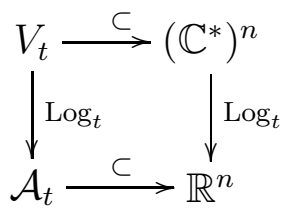

The limit of $\log _{t}\left(V_{t}\right) \subset \mathbb{R}^{n}$ coincides with the non-Archimedean amoeba $\mathcal{A} \subset \mathbb{R}^{n}$ and is an example of tropical variety by degeneration of complex varieties.

It was shown by Kapranov Kap00 that if $V \subset\left(K^{*}\right)^{n}$ is a hypersurface then $\mathcal{A}=\operatorname{Val}(V)$ depends only on the valuation of coefficients of the polynomial defining $V$. These valuations are elements of $\mathbb{T}$ and can be considered as coefficients of the tropical polynomial defining $\mathcal{A}$. 
The construction above gives a restricted class of tropical varieties. In particular, they are embedded to $\mathbb{R}^{n}=\left(\mathbb{T}^{*}\right)^{n}$. As the subject of this paper is tropical curves we won't give a general definition of higher-dimensional tropical varieties here (see [Mik06]). On the other hand, in this paper we define tropical curves intrinsically and study their inner geometry that does not depend on a particular embedding to an ambient space.

To conclude the introduction we give the definition of a particularly useful higherdimensional tropical variety.

Definition 1.1. The tropical projective space $\mathbb{T} \mathbb{P}^{n}$ consists of the classes of $(n+1)$ tuples of tropical numbers such that not all of them are equal to $-\infty$ with respect to the following equivalence relation. We say that

$$
\begin{gathered}
\left(x_{0}: \cdots: x_{n}\right) \sim\left(y_{0}: \cdots: y_{n}\right), \\
x_{j}, y_{j} \in \mathbb{T}, " \prod_{j=0}^{n} x_{j} "=\sum_{j=0}^{n} x_{j} \neq-\infty \text { and " } \prod_{j=0}^{n} y_{j} " \neq-\infty \text { if there exists } \lambda \in \mathbb{T}^{*} \text { such }
\end{gathered}
$$
that $x_{j}=y_{j}+\lambda, j=0, \ldots, n$.

Note that $\mathbb{T} \mathbb{P}^{n}$ contains $\mathbb{R}^{n}=\left(\mathbb{T}^{*}\right)^{n}$ : a point $\left(x_{1}, \ldots, x_{n}\right)$ corresponds to $\left(0: x_{1}\right.$ : $\left.\cdots: x_{n}\right)$. Thus we have an embedding

$$
\iota_{n}: \mathbb{R}^{n} \subset \mathbb{T P}^{n} .
$$

Also we have $n+1$ affine charts $\mathbb{T}^{n} \rightarrow \mathbb{T P}^{n}$, given by the (tropical) ratio with the $j$ th coordinate.

\section{SOME TROPICAL ALGEBRA}

2.1. Tropical modules. Recall that a commutative semigroup with zero is a set $V$ equipped with an arithmetic operation (called addition and denoted with "+") that is commutative, associative and such that there exists a neutral element (called zero in $V$ and denoted with $-\infty)$.

Definition 2.1. A tropical module or a $\mathbb{T}$-module $V$ is a commutative semigroup with zero equipped with a map $\mathbb{T} \times V \rightarrow V$ (called multiplication by a scalar) such that

- "c $c(v+w) "=$ " $c v+c w "$ for any $c \in \mathbb{T}$;

- "c(d(v))" = " $(c d) v "$ for any $c, d \in \mathbb{T}, v \in V$;

- " $0 v "=$ " $v "$ for the multiplicative unit $0 \in \mathbb{T}$ and any $v \in V$;

- if " $c v "=$ " $d v "$ for some $c, d \in \mathbb{T}, v \in V$ then either $c=d$ or $v=-\infty$.

A basic example of a tropical module is the free $n$-dimensional tropical module $\mathbb{T}^{n}$. The addition and multiplication by a scalar are the corresponding coordinatewise tropical operations. However, unlike the case with honest vector spaces over fields, there do exist other tropical modules. 
Example 2.2. Let $V_{n} \subset \mathbb{T}^{n}$ be the submodule generated by elements

$$
e_{1}=(-\infty, 0, \ldots, 0), e_{2}=(0,-\infty, 0, \ldots, 0), \ldots, e_{n}=(0, \ldots, 0,-\infty) .
$$

Elements of $V_{n}$ are linear combinations " $\sum_{j=1}^{n} c_{j} e_{j}$ " for some $c_{j} \in \mathbb{T}$.

It is easy to see that (at least as a topological space with topology induced from $\mathbb{T}^{n}=[-\infty,+\infty)^{n}$ ), the module $V_{n}$ is 2-dimensional yet it is distinct from $\mathbb{T}^{2}$ (or $\mathbb{T}^{k}$ with any other $k$ ). Indeed, suppose that $a_{j} \in \mathbb{T}$ and $a_{1} \geq a_{2} \geq \ldots \geq a_{n}$. Then

$$
\text { " } \sum a_{j} e_{j} "=\left(a_{2}, a_{1}, \ldots, a_{1}\right) .
$$

For algebraic considerations we need an intrinsic definition of dimension.

Definition 2.3. Let $V$ be a tropical module. We say that its dimension is smaller than $k$ if for any elements $v_{1}, \ldots, v_{k} \in V$ and any linear combination

$$
v=\sum_{j=1}^{k} c_{j} v_{j}
$$

$c_{j} \in \mathbb{T}$, the element $v$ can be presented as a tropical linear combination of a proper subset of $\left\{v_{1}, \ldots, v_{k}\right\}$.

We set the dimension to be equal to the maximal value of $k$ such that the dimension is not smaller than $k$.

Proposition 2.4. The dimension of $V_{n}$ (see Example 2.2) equals 2 regardless of $n$. Proof. Suppose that $v=\sum_{j=1}^{k} c_{j} v_{j}$. We saw that each $c_{j} v_{j}$ is parameterized by two numbers $a_{1}^{(j)} \geq a_{2}^{(k)} \in \mathbb{T}$. In the collection $c_{j} v_{j}$ we keep the vector $v_{j}$ with the maximal $a_{1}^{(j)}$ and another vector $c_{k} v_{k}$ with the maximal coordinate at the position of $a_{2}^{(j)}$.

Let us introduce another useful characteristic of a $\mathbb{T}$-module $V$. The rank of $V$ is the minimal number of generators of $V$. Note that even though the modules $V_{n}$ have the same dimension, they have different ranks.

Proposition 2.5. The dimension from Definition 2.3 coincides with the topological dimension of $V$.

Proof. Fixing $v_{1}, \ldots, v_{k}$ gives us a piecewise-linear continuous map $\mathbb{T}^{k} \rightarrow V$, $\left(c_{1}, \ldots, c_{n}\right) \mapsto " \sum_{j=1}^{k} c_{j} v_{j} "$. Suppose it is a surjection to $U \subset V$. If the topological dimension of its image is $d$ then $k \geq d$. On the other hand by the convexity argument $U$ has to be contained in the image of the union of the $d$-dimensional coordinate subspaces of $\mathbb{T}^{k}$. 
2.2. Projectivization of a tropical module. We may generalize the construction of $\mathbb{T P} \mathbb{P}^{n}$ by projectivizing any tropical module $V$, not necessarily a free module $\mathbb{T}^{n+1}$.

Definition 2.6. Let $V$ be a tropical module. Its projectivization $\mathbb{P}(V)$ consists of the classes of the non-zero elements of $v$ with respect to the following equivalence relation. We say that

$$
v \sim v^{\prime}
$$

if there exists $\lambda \in \mathbb{T}^{*}$ such that $v=" \lambda v^{\prime}$.

Clearly we have $\mathbb{P}\left(\mathbb{T}^{n+1}\right)=\mathbb{T P}^{n}$.

Example 2.7. The projectivization $\mathbb{P}\left(V_{n}\right)$ of the module from Example 2.2 is called $\Gamma_{n}$. It will serve as our local model for a tropical curve.

Note that by definition we have $\Gamma_{n} \subset \mathbb{T P}^{n-1}$.

\section{TROPICAL CURVES}

3.1. Definitions. Any affine-linear map $A: \mathbb{R}^{k} \rightarrow \mathbb{R}^{k^{\prime}}$ is a composition of a linear $\operatorname{map} L_{A}: \mathbb{R}^{k} \rightarrow \mathbb{R}^{k^{\prime}}$ and a translation. We call $A$ a $\mathbb{Z}$-affine map if its linear part $L_{A}$ is defined over $\mathbb{Z}$ (i.e. given by a matrix with integer entries) no matter what is its translational part.

Let $C$ be a connected topological space homeomorphic to a locally finite 1dimensional simplicial complex.

Definition 3.1. A complete tropical structure on $C$ is an open covering $\left\{U_{\alpha}\right\}$ of $C$ together with embeddings

$$
\phi_{\alpha}: U_{\alpha} \rightarrow \mathbb{T} \mathbb{P}^{k_{\alpha}-1}
$$

called the charts, $k_{\alpha} \in \mathbb{N}$, subject to the following conditions that must hold for any $\alpha$ and $\beta$.

- We have $\phi_{\alpha}\left(U_{\alpha}\right) \subset \Gamma_{k_{\alpha}}$.

- If $U^{\prime} \subset U_{\alpha}$ is an open subset then $\phi_{\alpha}\left(U^{\prime}\right)$ is open in $\Gamma_{k_{\alpha}} \subset \mathbb{T} \mathbb{P}^{k_{\alpha}-1}$.

- The "finite part" $\iota_{k_{\alpha}-1}^{-1} \circ \phi_{\alpha} \circ \phi_{\beta}^{-1} \circ \iota_{k_{\beta}-1}$ of the corresponding overlap maps is a restriction of a $\mathbb{Z}$-affine linear map $\mathbb{R}^{k_{\beta}-1} \rightarrow \mathbb{R}^{k_{\alpha}-1}$. Here we consider the map $\iota_{k_{\alpha}-1}^{-1} \circ \phi_{\alpha} \circ \phi_{\beta}^{-1} \circ \iota_{k_{\beta}-1}$ only where it is defined. The embedding $\iota_{n}: \mathbb{R}^{n} \rightarrow \mathbb{T P}^{n}$ is taken from (1).

- If $S \subset U_{\alpha}$ is a closed set in $C$ then $f(S) \cap \iota_{k_{\alpha}-1}\left(\mathbb{R}^{k_{\alpha}-1}\right)$ is a closed set in $\iota_{k_{\alpha}-1}\left(\mathbb{R}^{k_{\alpha}-1}\right)$.

The space $C$ equipped with a complete tropical structure is called a tropical curve. The genus of $C$ is its first Betti number $\operatorname{dim} H_{1}(C)$. We are especially interested in compact curves. E.g. the first two curves on Figure 1 are not compact as each of them has an open end. Meanwhile, it is easy to compactify these curves by adding a point at infinity for each open end. 

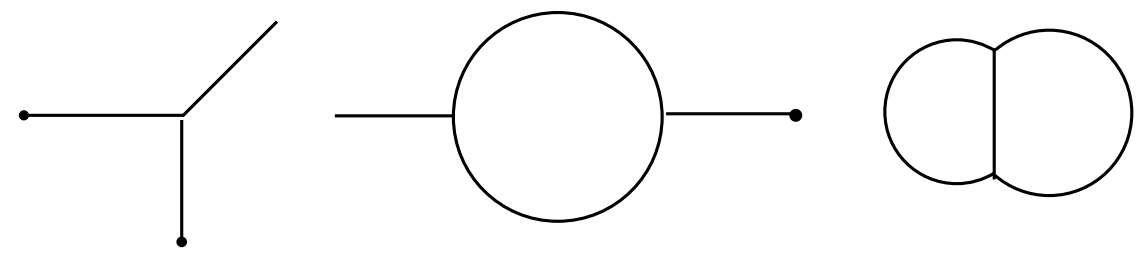

Figure 1. Examples of tropical curves of genus 0,1 and 2.

Clearly, the valence of a vertex of a 1-dimensional simplicial complex is a topological invariant. Furthermore, a point inside of an edge can be prescribed to be of valence 2 as it is possible to introduce a new 2-valent vertex at such point.

Definition 3.2. The finite part $C^{\circ}$ of $C$ is the complement of all 1 -valent vertices in $C$. The 1-valent vertices are called the endpoints of $C$.

Proposition 3.3. For any chart $\phi_{\alpha}: U_{\alpha} \rightarrow \mathbb{T P}^{k_{\alpha}-1}$ we have

$$
\phi_{\alpha}^{-1}\left(\iota_{k_{\alpha}-1}\left(\mathbb{R}^{n}\right)\right)=C^{\circ} \cap U_{\alpha} .
$$

This proposition follows from the last condition in Definition 3.1, the one that is responsible for the completeness of the tropical structure. In other words, the 1 -valent vertices must sit on the boundary part of $\mathbb{T}^{N}$.

Definition 3.4. Suppose that $C_{1}$ and $C_{2}$ are tropical curves. A continuous map

$$
f: C_{1} \rightarrow C_{2}
$$

is called tropical if for every $x \in C_{1}$ there exists an open neighborhood $U \ni x$ and charts $U_{\alpha} \supset U$ and $U_{\beta} \ni f(x), U_{\alpha} \subset C_{1}, U_{\beta} \subset C_{2}$, such that $\iota_{k_{\beta}} \circ \phi_{\beta} \circ f \circ \phi_{\alpha}^{-1} \circ \iota_{k_{\alpha}}$ is a restriction of a $\mathbb{Z}$-affine map $\mathbb{R}^{k_{\alpha}} \rightarrow \mathbb{R}^{k_{\beta}}$.

Tropical curves are called isomorphic if there exist mutually inverse tropical maps between them.

3.2. Equivalence of tropical curves. It turns out that some non-isomorphic tropical curves serve as two distinct models for the same variety. It is convenient to introduce an equivalence relation identifying such models.

Let $C$ be a tropical curve, $x \in C^{\circ}$ be a point in the finite part of $C$. Restricting to another chart if needed we can find a chart $\phi_{\alpha}: U_{\alpha} \rightarrow \mathbb{T P}^{k_{\alpha}-1}$ such that $\phi_{\alpha}(x)=$ $\left(1_{\mathbb{T}}: \cdots: 1_{\mathbb{T}}\right)=(0: \cdots: 0)$ is the "center" of the curve $\Gamma_{k_{\alpha}} \subset \mathbb{T P}^{k_{\alpha}-1}$.

The projection along the last coordinate defines a continuous map $\lambda_{k_{\alpha}}: \mathbb{T P}^{k_{\alpha}} \backslash$ $\{(-\infty: \cdots:-\infty: 0)\} \rightarrow \mathbb{T P}^{k_{\alpha}-1}$. The map

$$
\lambda_{\Gamma_{k_{\alpha}}}: \Gamma_{k_{\alpha}+1} \rightarrow \Gamma_{k_{\alpha}}
$$

obtained by restricting $\lambda_{k_{\alpha}}$ to $\Gamma_{k_{\alpha}+1}$ and extending it to $(-\infty: \cdots:-\infty: 0)$ is continuous. 
The map $\lambda_{\Gamma_{k_{\alpha}}}$ contracts the interval $[(0: \cdots: 0),(-\infty: \cdots:-\infty: 0)]$ to $(0: \cdots: 0)$ and is a homeomorphism to its image when restricted to $\Gamma_{k_{\alpha}+1} \backslash((0:$ $\cdots: 0),(-\infty: \cdots:-\infty: 0)]$. Let

$$
\tilde{U}_{\alpha}=\lambda_{\Gamma_{k_{\alpha}}}^{-1}\left(\phi_{\alpha}\left(U_{\alpha}\right)\right)
$$

We form $\tilde{C}=C_{x}$ by replacing the neighborhood $\tilde{U}_{\alpha}$ with $U_{\alpha}$. Clearly, the tropical structure naturally extends to the whole curve $\tilde{C}$. Topologically, $\tilde{C}$ is the result of gluing of a closed interval $[0,1]$ to $C$ by identifying $0 \in[0,1]$ with $x \in C$. Denote with $\tilde{x} \in \tilde{C}$ the point corresponding to $1 \in[0,1]$. (See [Mik06] for a more general procedure that can be used to define higher-dimensional tropical varieties.)

Definition 3.5. The map

$$
\tilde{C} \rightarrow C
$$

induced by (2) is called the elementary equivalence of tropical curves. Two curves $C_{1}$ and $C_{2}$ are called tropically equivalent if they can be connected with a sequence of elementary equivalences (in any direction).

This equivalence relation provides a convenient tool for working with curves with marked points. If $x \in C^{\circ}$ is in the affine part then we may replace $C$ with $\tilde{C}=C_{x}$ and $x$ with $\tilde{x}$. In this way we may replace a curve $C$ with a collection of marked points on it with an equivalent curve $\bar{C}$, so that all the marked points will be the endpoints of $\bar{C}$. This trick turns out to be very useful for the setup of the GromovWitten theory, cf. Mik.

Here we would just like to note that putting marked points to the end of the equivalent curve can be used to puncture a tropical curve. If we need to remove a point $x \in C^{\circ}$ then first we replace $(C, x)$ with an equivalent pair $(\tilde{C}, \tilde{x})$ and then treat $\tilde{C} \backslash \tilde{x}$ as the result of puncturing $C$ at $x$. Note that $\tilde{C} \backslash \tilde{x}$ is a tropical curve while $C \backslash x$ itself is not. Furthermore, the contraction map (3) can be considered as a morphism analogue of a Zariski-open set (in the Grothendieck topology style), see [Mik] for details.

3.3. Tropical curves as metric graphs. A tropical curve turns out to carry the same information as the so-called metric graph. Recall that the edges adjacent to one-valent vertices of a graph are called leaves. Other (non-leaf) edges are called inner. A metric graph (cf. e.g. [BHV01]) is a graph equipped with finite positive length for all its inner edges. The leaves of a metric graph are prescribed the (positive) infinite length.

Let $\Gamma$ be a connected finite graph and $\mathcal{V}_{1}(\Gamma)$ be its 1-valent vertices (or endpoints). We may consider

$$
\Gamma^{\circ}=\Gamma \backslash \mathcal{V}_{1}(\Gamma)
$$


If $\Gamma$ is a metric graph then $\Gamma^{\circ}$ is a complete metric space (with an inner metric). Vice versa, a complete metric space homeomorphic to $\Gamma^{\circ}$ for some finite graph $\Gamma$ defines a metric graph.

Recall that if $X$ is any topological space one can consider its maximal compactification $\bar{X}$ by adding a point "at infinity" for each end of $X$. Clearly, $\Gamma$ and $\bar{\Gamma}^{\circ}$ are homeomorphic if $\Gamma$ is a finite graph.

Proposition 3.6. There is a natural 1-1 correspondence between compact tropical curves and metric graphs.

Proof. Let $L \subset \mathbb{R}^{k}$ be a line of a rational slope and let $\xi \in \mathbb{Z}^{k}$ be a primitive (i.e. not divisible by an integer) vector parallel to $L$. Define the distance between points $x, y \in L$ to be $\frac{\|x-y\|}{\|\xi\|}$, where $\|$. $\|$ stands for any norm in the vector space $\mathbb{R}^{k}$. This metric is preserved by the overlap maps $\left.\phi_{\alpha} \circ \phi_{\beta}^{-1}\right|_{\phi_{\beta}\left(U_{\alpha} \cap U_{\beta}\right) \cap \mathbb{R}^{k} \beta}$. This gives us a natural inner metric on a tropical curve.

Vice versa, if $C$ is a metric graph then we set the metric-preserving charts to $\mathbb{R}$ for the interior of the edges; charts to $\mathbb{T}$ for small neighborhoods of 1 -valent vertices and isometric embedding charts to $\Gamma_{k}$ for small neighborhoods of multivalent vertices.

3.4. Regular and rational functions on curves. By the very definition a tropical curve $C$ is a topological space homeomorphic to graphs equipped with an additional geometric structure which we call the tropical structure. It allows one to define a sheaf of regular function on $C$. We call a function $\mathbb{R}^{n} \rightarrow \mathbb{R} \subset \mathbb{T} \mathbb{Z}$ affine-linear if it is obtained from a function $\mathbb{R}^{n} \rightarrow \mathbb{R}$ linear over $\mathbb{Z}$ by adding a real constant.

Let $U \subset C$ be an open set.

Definition 3.7. A function $f: U \rightarrow \mathbb{T}$ is called regular if for any $x \in U$ there exists a chart

$$
\begin{gathered}
\phi_{\alpha}: U_{\alpha} \rightarrow \mathbb{T}^{n} \subset \mathbb{T} \mathbb{P}^{n}, \\
n=k_{\alpha}-1, U_{\alpha} \ni x, \text { a tropical polynomial } g: \mathbb{T}^{n} \rightarrow \mathbb{T}, \\
g\left(x_{1}, \ldots, x_{n}\right)=" \sum_{j_{1}, \ldots, j_{n}} a_{j_{1} \ldots j_{n}} x_{1}^{j_{1}} \ldots x_{n}^{j_{n}} "=\operatorname{Max}_{j_{1}, \ldots, j_{n}}\left(a_{j_{1} j_{j_{n}}}+j_{1} x_{1}+\cdots+j_{n} x^{n}\right)
\end{gathered}
$$

such that the function $f \circ \phi_{\alpha}^{-1}-g$ (which is defined on $\phi_{\alpha}(U)$ ) restricted to $\mathbb{R}^{n} \subset \mathbb{T}^{n}$ is a $\mathbb{Z}$ affine-linear function. Here $\mathbb{T}^{n} \subset \mathbb{T} \mathbb{P}^{n}$ is the embedding given by $\left(x_{1}, \ldots, x_{n}\right) \mapsto$ $\left(0: x_{1}: \cdots: x_{n}\right)$. If the function $g$ may be chosen to be a constant then $f$ is called an affine-linear function on $U$.

A function $h: U \rightarrow \mathbb{T}$ is called rational if there exist two regular functions $f_{1}, f_{2}: U \rightarrow \mathbb{T}$ such that

$$
h=" \frac{f_{1}(x)}{f_{2}(x)} "=f_{1}(x)-f_{2}(x)
$$

for any $x \in U \cap C^{\circ}$. 
Clearly, regular functions form a sheaf of tropical algebras on $C$. The same holds for rational functions. The sheaf of regular functions is called the structure sheaf and is denoted with $\mathcal{O}_{C}^{\mathbb{T}}$.

3.5. Projective tropical curves, tropical curves in $\mathbb{R}^{n}$ and in other higherdimensional tropical varieties. Linear morphisms maybe used to embed or immerse tropical curves into tropical toric varieties that arise as compactifications of $\mathbb{R}^{n}=\left(\mathbb{T}^{\times}\right)^{n}$. Our main example of such compactification is $\mathbb{T} \mathbb{P}^{n}$.

Let $f: C \rightarrow \mathbb{T}$ be an affine-linear function. For every edge $E \subset C$ we may define the slope of $f$ once we fix an orientation of $E$. Indeed if $\xi$ is a primitive tangent vector consistent with the choice of the orientation then the slope is just the partial derivative $\frac{\partial f}{\partial \xi}$. If we do not fix the orientation of $E$ then the slope is only defined up to the sign.

Definition 3.8. A map $C \rightarrow \mathbb{T}^{n}$ is called a linear morphism if it is given in coordinates by affine-linear functions. A map $C \rightarrow \mathbb{T} \mathbb{P}^{n}$ is called a linear morphism if locally (with respect to the charts $\mathbb{T}^{n} \subset \mathbb{T P}^{n}$ ) it is given by linear morphisms.

Remark. This definition agrees with a more general definition given in Mik06. It is easy to show that every map $C \rightarrow \mathbb{T P}^{n}$ locally given in coordinates by regular functions admits a resolution by a linear morphism $\tilde{C} \rightarrow \mathbb{T}^{n}$ for an equivalent tropical curve $\tilde{C}$ with a contraction $\tilde{C} \rightarrow C$.

Let $C$ be a compact tropical curve and $h: C \rightarrow \mathbb{T} \mathbb{P}^{n}$ be a linear morphism. The image $h(E)$ of an edge $E \subset C$ is either a point (if the slope of all coordinates is zero) or a straight interval with a rational slope (as the slopes of all coordinates are integers). We define the weight $w(E)$ as the GCD of the coordinate slopes of $E$. The collection of the slopes of all coordinates gives the weight vector $\xi_{E} \in \mathbb{Z}^{n}$ (defined up to a sign unless we specify the orientation of $E$ ). The ratio $\frac{\xi_{E}}{w(E)}$ is a primitive integer vector parallel to $E$.

The image $h(E)$ is contained in the finite part $\mathbb{R}^{n} \subset \mathbb{T} \mathbb{P}^{n}$ if and only if both vertices adjacent to $E$ have valence greater than 1 . All 1-valent vertices of $C$ are mapped to the boundary part $\mathbb{T} \mathbb{P}^{n} \backslash \mathbb{R}^{n}$. Let $P \subset C$ be a vertex and $E_{1}, \ldots, E_{k}$ are the edges adjacent to $P$. If $k>1$ then $h(P) \in \mathbb{R}^{n}$ and $\sum_{j=1}^{k} \xi_{k}=0$.

Recall that a leaf of a graph is an edge adjacent to a 1-valent vertex. The degree of a projective curve is the number of leaves (counted with weight) adjacent to any of the $(n+1)$ components of the boundary divisor $\mathbb{T} \mathbb{P}^{n} \backslash \mathbb{R}^{n}$. This definition agrees with the one given in Mik05]. Note that linear morphisms of different curves may have the same image, see Figure 2 for an example.

If $X \subset \mathbb{T} \mathbb{P}^{n}$ is a projective tropical variety (see [Mik06]) then we define a (parameterized) tropical curve in $X$ as a linear morphism $C \rightarrow \mathbb{P P}^{n}$ such that its image is contained in $X$. Note that to embed $X$ to a projective space we often need to pass to an equivalent model of $X$. 


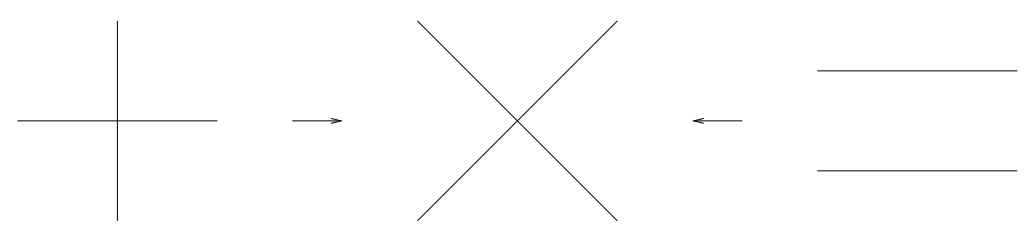

Figure 2. A cross figure (in the center) obtained as the image of two different linear morphisms.

\section{LINE BUNDLES ON CURVES}

4.1. Tangent vectors and $\mathbf{1}$-forms. Let $\xi$ be a primitive integer vector tangent to an interval in $\phi_{\beta}\left(U_{\alpha} \cap U_{\beta}\right) \subset \mathbb{R}^{k_{\beta}}$. Note that the overlap maps $\left.\phi_{\alpha} \circ \phi_{\beta}^{-1}\right|_{\phi_{\beta}\left(U_{\alpha} \cap U_{\beta}\right) \cap \mathbb{R}^{k_{\beta}}}$ takes $\xi$ to a primitive integer tangent vector to an interval in $\phi_{\alpha}\left(U_{\alpha} \cap U_{\beta}\right) \subset \mathbb{R}^{k_{\alpha}}$ (since the overlap map admits a $\mathbb{Z}$-affine inverse). Thus we have a well-defined notion of a primitive tangent vector to a point $p \in C$.

If $p$ is a point in the interior of an edge then it has two primitive tangent vectors $\xi_{1}$ and $\xi_{2}$. We have $\xi_{2}=-\xi_{1}$. An integer vector tangent to $C$ at $p$ is an integer multiple of $\xi_{1}\left(\right.$ or $\left.\xi_{2}\right)$.

If $p$ is a vertex of $C$ then we can distinguish between outward and inward primitive tangent vectors at $p$. If $p$ is a $k$-valent vertex then it has $k$ outward tangent vectors $\xi_{1}, \ldots, \xi_{k}$ with

$$
\sum_{j=1}^{k} \xi_{j}=0
$$

(the curve $C$ is an abstract curve now, but this equality makes sense in any affine chart of $C$ ). An integer vector tangent to $C$ at $p$ is an integer linear combination of $\xi_{1}, \ldots, \xi_{k-1}$. It defines an integer vector in $\mathbb{R}^{k_{\alpha}}$ for any chart $U_{\alpha} \ni p$.

Note that there is no difference between a 2-valent vertex and an interior point of an edge. Both of them have affine charts to $\mathbb{R}$. At our convenience we may introduce extra 2-valent vertices by subdividing an edge or do the opposite operation.

4.2. Universal covering. Let us fix $g$ break points $z_{1}, \ldots, z_{g}$ in the interiors of the edges of $C$ such that $T:=C \backslash\left\{z_{1}, \ldots, z_{g}\right\}$ is a tree. Then $T$ is connected and has $g$ pairs of "break" ends. We will order each pair $z_{i}^{ \pm}$by a primitive tangent vector $\nu_{i}$ at $z_{i}: \nu_{i}$ points outward at $z_{i}^{+} \in \bar{T}$ and inward at $z_{i}^{-} \in \bar{T}$.

The universal covering of the graph $C$ is an infinite tree and we will later use $\bar{T}$ as its fundamental domain.

4.3. Divisors. A divisor on $C$ is a formal finite sum of points $D=\sum a_{i} p_{i}$, where all $a_{i}$ 's are integers and $p_{i} \in C$. The set of all divisors form an abelian group which we will denote by $\operatorname{Div}(C)$. Given a rational function $f$ on an open subset $U \subset C$ 

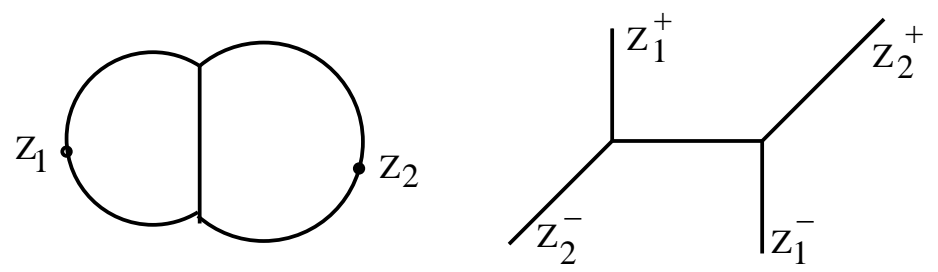

Figure 3. A fundamental domain $\bar{T}$ of the universal covering.

one can consider the following formal sum:

$$
(f):=\sum_{p \in U}\left(\sum_{i=1}^{k(p)} \frac{\partial f}{\partial \xi_{i}}(p)\right) p .
$$

If $U=C$ then the sum in the right hand side is finite and thus defines a divisor on $C$. Such divisors of global rational functions are called principal. We say that two divisors are linearly equivalent: $D_{1} \sim D_{2}$, if $D_{1}-D_{2}$ is principal.
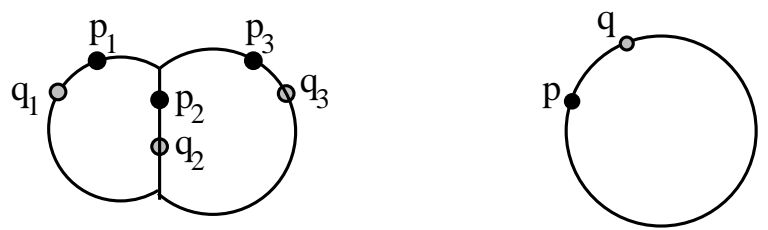

FiguRE 4. Linear equivalence: $p_{1}+p_{2}+p_{3} \sim q_{1}+q_{2}+q_{3}$, but $p \nsim q$.

\subsection{Line bundles.}

Definition 4.1. A line bundle on $C$ is an $\mathcal{O}^{*}$-torsor, where the sheaf of affine functions $\mathcal{O}^{*}$ acts on the structure sheaf by tropical multiplication. Equivalently a line bundle $L$ is a topological space together with the following data:

- A continuous projection $\pi: L \rightarrow C$ with fibers $\mathbb{T}$.

- Every point $p \in C$ has an open neighborhood $U_{p} \ni p$ and families of trivialization maps $\{\phi\}_{V}: \pi^{-1}(V) \cong V \times \mathbb{T}$ for any open $V \subset U_{p}$, which are the restrictions from $\{\phi\}_{U_{p}}: \pi^{-1}\left(U_{p}\right) \cong U_{p} \times \mathbb{T}$, and such that any two trivializations $\phi_{1}, \phi_{2} \in\{\phi\}_{V}$ differ by (tropical multiplication by) an affine linear function in $V$.

Given a sufficiently fine open covering $\left\{U_{\alpha}\right\}$ of $C$ a line bundle can be specified by transition functions $f_{\alpha \beta}: U_{\alpha} \cap U_{\beta} \rightarrow \mathbb{R}$ between trivializations. The functions $f_{\alpha \beta}$ are affine linear and satisfy the usual cocycle condition. Another choice of trivializations will result in changing the cocycle by a coboundary. A continuous map $L_{1} \rightarrow L_{2}$ which respects the projection and local trivialization up to affine functions is an isomorphism of line bundles. Thus, as in the classical geometry, the isomorphism 
classes of line bundles are parameterized by the first Čech cohomology $H^{1}\left(C, \mathcal{O}^{*}\right)$. This is a group and we will refer to it as the Picard group $\operatorname{Pic}(C)$.

Note that equivalent tropical curves have the same Picard group as the restriction of a line bundle on a (contractible) infinite edge is necessarily trivial.

4.5. Sections. Given an open subset $U \subset C$ a section $s: U \rightarrow \pi^{-1}\left(U_{p}\right)$ is regular, respectively rational, if for any open $V \subset U$ and a trivialization $\phi: \pi^{-1}(V) \cong V \times \mathbb{T}$, $s$ becomes a regular, resp. rational, function on $V$. The notion does not depend on trivializations. Neither does the formal sum in (4). Thus a global rational section of $L$ defines a divisor on $C$. There is the usual correspondence between line bundles and divisors up to equivalence:

Proposition 4.2. Every divisor defines a line bundle together with its rational section. This section is defined uniquely up to adding a constant (i.e. up to tropical multiplication).

Conversely, every line bundle $L$ has a rational section. The divisors of any two rational sections of $L$ are linearly equivalent.

Proof. Let $D=\sum a_{i} p_{i}$ be a divisor. We can cover $C$ by open sets $U_{i}$ so that each point $p_{i}$ is contained in a unique $U_{i}$. Then for each $U_{i}$ we choose a rational function $f_{i}$ whose order of zero (pole) at $p_{i}$ is $a_{i}$. The incompatibilities over the intersections define a Čech cocycle with values in affine linear functions. Hence we get a line bundle. The rational section is given by the collection $\left\{f_{i}\right\}$.

Conversely, given a line bundle $L$ we can choose a small open interval $N_{i}=$ $\left(a_{i}, b_{i}\right) \ni z_{i}$ for every break point $z_{i}$. Then $L$ can be trivialized over $C \backslash \cup N_{i}$ and over each of the $N_{i}$. Thus any rational function $f$ gives a section of $L$ on $C \backslash \cup N_{i}$. To extend $f$ to a section over $C$ one needs integral PL functions $f_{i}$ on the $N_{i}$ equal to given affine linear functions near the ends $a_{i}, b_{i}$. This is always possible. The resulting divisor consists of the divisor of $f$ on $C \backslash \cup N_{i}$ and of the divisors of the $f_{i}$ over each $N_{i}$.

For a divisor $D=\sum a_{j} p_{j}$ we define its degree in the usual way: $\operatorname{deg}(D):=\sum a_{i}$. We will say that the corresponding line bundle $L(D)$ has degree $d$ as well. The groups of degree $d$ divisors and line bundles will be denoted by $\operatorname{Div}^{d}(C)$ and $\operatorname{Pic}^{d}(C)$ respectively. Now we can reformulate Proposition 4.2.

Corollary 4.3. For every $d$ there is a canonical isomorphism $\operatorname{Pic}^{d}(C) \cong \operatorname{Div}^{d}(C) / \sim$.

The degree of a line line bundle can be calculated from the following proposition.

Proposition 4.4. Let $U$ be a connected open set in a tropical curve with boundary $\partial U=\left\{z_{1}, \ldots, z_{k}\right\}$ and $\nu_{1}, \ldots, \nu_{k}$ - the corresponding outward primitive tangent vectors. Let $f$ be a rational function on $U$ with divisor $D=\sum a_{j} p_{j}$. Then

$$
\operatorname{deg}(D)=\sum_{i=1}^{k} \frac{\partial f}{\partial \nu_{i}}\left(z_{i}\right)
$$


Proof. We observe that the formula is additive when gluing pieces of the curve at the points others than $p_{i}$. Note also, that the formula trivially holds for affine linear functions. On the other hand at the points $p_{i}$ it is just the definition of the order $a_{i}=\sum_{j=1}^{k(p)} \frac{\partial f}{\partial \xi_{j}}\left(p_{i}\right)$.

Corollary 4.5. A principal divisor on a compact curve has degree zero.

4.6. The space of global sections of a line bundle. Principal divisors can be handy in describing the space of global regular sections of a given line bundle. Let $D_{0}$ be a divisor and let $s_{0}$ be the corresponding rational section (unique up to an additive constant) of the line bundle $L:=L\left(D_{0}\right)$. Then any other rational section $s$ of $L$ is given by $s=s_{0}+f$, where $f$ is a rational function. The section $s$ is regular if the divisor $D_{0}+(f)$ is effective (i.e. all coefficients are positive).

The space of global sections $\Gamma(C, L)$ has the structure of an $\mathbb{T}$-module. Given $s_{1}, s_{2} \in \Gamma(C, L)$ one can take their tropical sum $\max \left\{s_{1}, s_{2}\right\}$ by choosing trivializations over open sets $U \subset C$ and considering $s_{1}, s_{2}$ as functions on $U$. The result is a section of the same bundle and it is independent of the trivialization. Adding a constant to a section is well defined too.

\section{TORI AND POLARIZATIONS}

A tropical torus $X$ is the quotient $\mathbb{R}^{n} / \Lambda$, where $\mathbb{R}^{n}$ is considered with an integral affine structure (that is with a fixed integral lattice $\mathbb{Z}^{n} \subset \mathbb{R}^{n}$ ) and $\Lambda \subset \mathbb{R}^{n}$ is a full rank lattice. We will denote by $\left(\mathbb{R}^{n}\right)^{*}$ the dual space with $\left(\mathbb{Z}^{n}\right)^{*}$ and $\Lambda^{*}$ - the dual integral lattices to $\mathbb{Z}^{n}$ and $\Lambda$.

5.1. Line bundles and polarizations. We can extend the most of our definitions and results on divisors and line bundles defined on curves to the case of tori. A line bundle is defined by an element in $H^{1}\left(X, \mathcal{O}^{*}\right)$, where $\mathcal{O}^{*}=$ Aff is the sheaf of affine linear functions on $X$. By induction on dimension of $X$ it is easy to see that any line bundle has a rational section.

Now consider a short exact sequence:

$$
0 \longrightarrow \mathbb{R} \longrightarrow \text { Aff } \longrightarrow T_{\mathbb{Z}}^{*} \longrightarrow 0,
$$

where $\mathbb{R}$ is locally constant sheaf and the sheaf $T_{\mathbb{Z}}^{*} \cong\left(\mathbb{Z}^{n}\right)^{*}$ has the meaning of locally constant sections of the integral cotangent bundle. The induced map of the long exact sequence

$$
c: H^{1}(X, \text { Aff }) \longrightarrow H^{1}\left(X, T_{\mathbb{Z}}^{*}\right)
$$

is called the Chern class map. A choice of a class $[c] \in H^{1}\left(X, T_{\mathbb{Z}}^{*}\right) \cong \Lambda^{*} \otimes\left(\mathbb{Z}^{n}\right)^{*}$ which is in the image of the Chern class map is called a polarization of $X$. Using the natural isomorphism $\Lambda^{*} \otimes\left(\mathbb{Z}^{n}\right)^{*} \cong \operatorname{Hom}\left(\Lambda,\left(\mathbb{Z}^{n}\right)^{*}\right)$ one can think of $[c]$ either as a bilinear form on $\mathbb{R}^{n}$ or as a map $[c]: \Lambda \rightarrow\left(\mathbb{Z}^{n}\right)^{*}$. 
We claim that the set of polarization classes is formed by the maps $\Lambda \rightarrow\left(\mathbb{Z}^{n}\right)^{*}$ which are symmetric as bilinear forms on $\mathbb{R}^{n}$. To see this we analyze the coboundary map in the induced long exact sequence:

$$
\delta: H^{1}\left(X, T_{\mathbb{Z}}^{*}\right) \longrightarrow H^{2}(X, \mathbb{R}) .
$$

By identifying $H^{1}\left(X, T_{\mathbb{Z}}^{*}\right) \cong \Lambda^{*} \otimes\left(\mathbb{Z}^{n}\right)^{*}$ and $H^{2}(X, \mathbb{R}) \cong \wedge^{2}\left(\mathbb{R}^{n}\right)^{*}$ one can deduce that $\delta$ is the restriction of the skew-symmetrization map $\left(\mathbb{R}^{n}\right)^{*} \otimes\left(\mathbb{R}^{n}\right)^{*} \rightarrow \wedge^{2}\left(\mathbb{R}^{n}\right)^{*}$.

Remark. For a general affine manifold the coboundary map $\delta$ is given by a wedge product with some characteristic class $\rho$ which takes values in the cohomology with coefficients in the tangent local system $T$. This so called radiance obstruction class has the meaning of the translational part of the monodromy representation (cf. KS01, Section 2.2). In our case the local system $T$ is trivial because the linear part of the monodromy on the torus is trivial. Thus, $H^{1}(X, T)$ can be canonically identified with $\left(\mathbb{R}^{n}\right)^{*} \otimes \mathbb{R}^{n}$, and $\rho$ is the identity element there.

We can also describe the set of line bundles with a given Chern class. Notice that the map $H^{0}(X, \mathbb{R}) \rightarrow H^{0}(X$, Aff $)$ is an isomorphism. Hence $H^{0}\left(X, T_{\mathbb{Z}}^{*}\right) \rightarrow H^{1}(X, \mathbb{R})$ is the natural inclusion $\left(\mathbb{Z}^{n}\right)^{*} \hookrightarrow\left(\mathbb{R}^{n}\right)^{*}$. Thus the Picard group of $X$ with a fixed Chern class can be identified with the dual torus $\left(\mathbb{R}^{n}\right)^{*} /\left(\mathbb{Z}^{n}\right)^{*}$.

If the quadratic form defined by $[c]$ is positive definite we call $\mathbb{R}^{n} / \Lambda$ a (polarized) tropical Abelian variety. The index of the polarization $[c]$ is the index of the image $[c](\Lambda)$ in $\left(\mathbb{Z}^{n}\right)^{*}$. We call a polarization principal if it has index one, that is, if the $\operatorname{map}[c]: \Lambda \rightarrow\left(\mathbb{Z}^{n}\right)^{*}$ is an isomorphism.

Theorem 5.1. Let $L$ be a line bundle on $X=\mathbb{R}^{n} / \Lambda$ which defines a principal polarization. Then the space of sections $\Gamma(X, L)$ is one dimensional.

Proof. Suppose $L$ has the Chern class $[c]: \Lambda \rightarrow\left(\mathbb{Z}^{n}\right)^{*}$. A global section $\Theta$ of $L$ can be viewed as a convex PL function on $\mathbb{R}^{n}$ subject to some quasi-periodicity condition:

$$
\Theta(x+\mu)=\Theta(x)+\langle[c](\mu), x\rangle+\beta(\mu), \quad \text { for any } \mu \in \Lambda .
$$

By applying quasi-periodicity twice we can see that $\beta$ has to be quadratic. Namely,

$$
\beta\left(\mu_{1}+\mu_{2}\right)=\beta\left(\mu_{1}\right)+\beta\left(\mu_{2}\right)+[c]\left(\mu_{1}, \mu_{2}\right) .
$$

That is, $\beta(\lambda)=\frac{1}{2}[c](\lambda, \lambda)+$ linear part. The linear part is responsible for shifts of the line bundle.

On the other hand, as a convex function $\Theta(x)$ is completely determined by its Legendre transform $\hat{\Theta}:\left(\mathbb{Z}^{n}\right)^{*} \rightarrow \mathbb{R}$. Now we show that a value of $\hat{\Theta}(0)$ determines value of $\hat{\Theta}$ at any point $m \in\left(\mathbb{Z}^{n}\right)^{*}$ which is in the image of $[c]$. Say $m=[c](\mu)$ for some $\mu \in \Lambda$, then

$$
\hat{\Theta}(-m)=\max _{x \in \mathbb{R}^{n}}\{\langle-[c](\mu), x\rangle-\Theta(x)\}=\max _{x \in \mathbb{R}^{n}}\{-\Theta(x)+\beta(\mu)\}=\hat{\Theta}(0)+\beta(\mu) .
$$


For a principal polarization, performing the inverse of the Legendre transform gives

$$
\Theta(x):=\max _{m \in\left(\mathbb{Z}^{n}\right)^{*}}\{\langle m, x\rangle-\hat{\Theta}(m)\}=\max _{\lambda \in \Lambda}\{\langle-[c](\lambda), x\rangle-\hat{\Theta}(0)-\beta(-\lambda)\} .
$$

This is the unique section of $L$ determined upto a additive constant by $\hat{\Theta}(0)$.

Remark. For a general tropical Abelian variety, a section of a positive line bundle $L$, thought of the quasi-periodic PL function $\Theta$ on $\mathbb{R}^{n}$, is completely determined by specifying values of its Legendre transform $\hat{\Theta}$ on every representative in the quotient $\left(\mathbb{Z}^{n}\right)^{*} /[c](\Lambda)$. Thus, in general, the dimension of $H^{0}(X, L)$ is given by the index of the polarization.

5.2. Theta functions. The unique (up to constant) section from Theorem 5.1 above will be the tropical analog of the classical Riemann's theta function. Given a full rank lattice $\Lambda$ in $\mathbb{R}^{n}$ and a positive definite symmetric bilinear form $Q$ : $\mathbb{R}^{n} \otimes \mathbb{R}^{n} \rightarrow \mathbb{R}$ we define

$$
\Theta(x):=\max _{\lambda \in \Lambda}\left\{Q(\lambda, x)-\frac{1}{2} Q(\lambda, \lambda)\right\}, \quad x \in \mathbb{R}^{n} .
$$

The maximum here always exists since $Q$ is positive definite form. From the definition we can readily see that $\Theta(x)$ is an odd function: $\Theta(-x)=\Theta(x)$. It also satisfies the following functional equation:

Lemma 5.2. $\Theta(x+\mu)=\Theta(x)+Q(\mu, x)+\frac{1}{2} Q(\mu, \mu)$, for any $\mu \in \Lambda$.

Proof. This calculation is in a sense "dual" to the the proof of Theorem 5.1. By considering effect of the translation on each term we have:

$$
\begin{aligned}
& Q(\lambda, x+\mu)-\frac{1}{2} Q(\lambda, \lambda) \\
& =Q(\lambda-\mu, x)+Q(\mu, x)+Q(\lambda, \mu)-\frac{1}{2} Q(\lambda-\mu, \lambda-\mu)-Q(\mu, \lambda)+Q(\mu, \mu) \\
& \quad=Q(\lambda-\mu, x)-\frac{1}{2} Q(\lambda-\mu, \lambda-\mu)+Q(\mu, x)+\frac{1}{2} Q(\mu, \mu) .
\end{aligned}
$$

Relabeling the terms and combining them into the tropical sum completes the proof.

The theta function above can be defined on $\mathbb{R}^{n}$ for an arbitrary positive symmetric bilinear form $Q$. But it is regular (holomorphic) in the tropical sense (i.e., with integral slopes) only if the form $Q$ is integral in the sense that the image of the induced map $\tilde{Q}: \Lambda \rightarrow\left(\mathbb{R}^{n}\right)^{*}$ ends up being inside the lattice $\left(\mathbb{Z}^{n}\right)^{*}$. From now on we restrict our attention to the integral forms $Q$ only. This is when the theta function defines a polarization on the tropical torus $\mathbb{R}^{n} / \Lambda$. Moreover, we will be primarily interested in principal polarizations. 
A tropical hypersurface in $\mathbb{R}^{n}$ defined by the corner locus of the theta function gives a honeycomb-like periodic decomposition of $\mathbb{R}^{n}$ which is dual to the triangulation of $\left(\mathbb{R}^{n}\right)^{*}$ with vertices in $\left(\mathbb{Z}^{n}\right)^{*}$ induced by the Legendre transform $\hat{\Theta}(m)$ (cf. Fig 5). The principal polarizations can be geometrically characterized by the fact
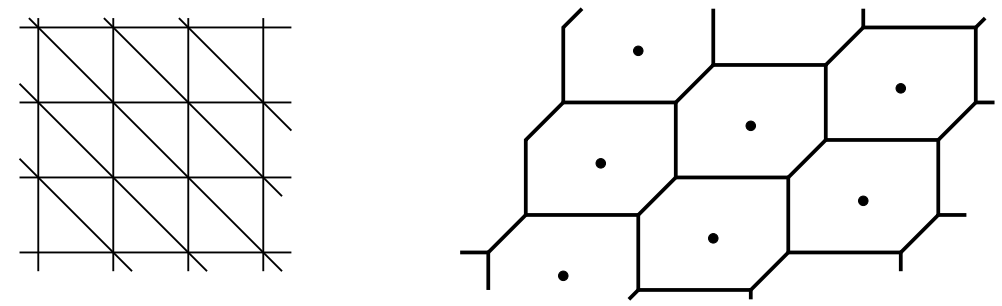

Figure 5. $\hat{\Theta}(m)$-induced triangulation of $\left(\mathbb{R}^{n}\right)^{*}$ and a corresponding $\Lambda$-periodic theta divisor.

that the volume of each $n$-cell has to be equal to the affine volume of the torus $\mathbb{R}^{n} / \Lambda$, which, in turn, is equal to $\operatorname{det} \tilde{Q}$.

The theta function can be considered as a section of the polarization line bundle $L$ on $X$ whose corresponding Cech 1-cocycle is defined by the "automorphy factors" $Q(\mu, x)+\frac{1}{2} Q(\mu, \mu)$ in the functional equation. The corner locus of the theta function descends to the quotient torus $X$ and form the theta divisor $[\Theta] \subset X$.

\section{Abel-JACOBi TheOrem}

6.1. Tropical Jacobian. A 1-form $\omega$ on $C$ is a section of the real cotangent local system $T^{*}$ on $C$ defined by the following exact sequence of sheaves:

$$
0 \longrightarrow \mathbb{R} \longrightarrow \operatorname{Aff}_{\mathbb{R}} \longrightarrow T^{*} \longrightarrow 0
$$

where the sheaf $\operatorname{Aff}_{\mathbb{R}}$ stands for affine functions with any real (not necessarily integral) slope. In particular, at the vertices $p$ a 1-form $\omega$ must have

$$
\sum_{i=1}^{v(p)} \omega\left(\xi_{i}\right)=0
$$

where $v(p)$ is the valence of $p$ and the $\xi_{i}$ 's are the primitive integral vectors at $p$.

Remark. The fact that the forms have to be constant on the edges of the graph can be interpreted in the degeneration picture as follows. The regular forms on complex curves which survive in the limit are of the form $\frac{\alpha}{2 \pi i} \frac{d z}{z}$ on long cylinders (edges to be). The coefficient $\alpha$ would be the value of the limiting form $\omega$ on the primitive vector tangent to the edge. The balancing condition (9) is then just the fact that the sum of residues of a rational form on $\mathbb{P}^{1}$ (a vertex to be) is zero. 
Denote by $\Omega(C)$ the space of global 1 -forms on $C$. Then by considering the values of the forms on the primitive vectors at the $g$ break points $z_{1}, \ldots, z_{g}$ we may identify $\Omega(C)$ with the $g$ dimensional $\mathbb{R}$-vector space. Integer valued forms constitute a full rank lattice $\Omega_{\mathbb{Z}}(C)$ inside $\Omega(C)$.

Given a path $\gamma$ in $C$, any 1-form $\omega$ on $C$ pulls back to a locally constant classical (old-fashioned) 1-form on an interval minus finite number of points. So that the integral $\int_{\gamma} \omega \in \mathbb{R}$ is well defined.

Let $\Omega(C)^{*}$ be the vector space of $\mathbb{R}$-valued linear functionals on $\Omega(C)$. Then the integral cycles $H_{1}(C, \mathbb{Z})$ form a lattice in $\Omega(C)^{*}$ by integrating over them. As in the classical complex geometry we define the Jacobian of the curve $C$ to be

$$
J(C):=\Omega(C)^{*} / H_{1}(C, \mathbb{Z}) \cong \mathbb{R}^{g} / \Lambda .
$$

Note that the $\mathbb{R}^{g}$ in the right hand side is naturally endowed with a $\mathbb{Z}$-structure: the lattice $\mathbb{Z}^{g} \subset \mathbb{R}^{g}$ is identified with the integer valued functionals on $\Omega_{\mathbb{Z}}(C)$. The $\mathbb{Z}^{g}$ is generated by the $g$ evaluation functionals $\omega \mapsto \omega\left(\eta_{i}\right)$ at the break points $z_{1}, \ldots, z_{g}$.

The metric on $C$ defines a symmetric bilinear form $Q$ on the space of paths in $C$ by setting $Q(\ell, \ell):=$ length $(\ell)$ for a simple (i.e., not self-intersecting) path $\ell$ and extending it to any pair of paths bilinearly. Since 0 in cohomology $H_{1}(C, \mathbb{Z})$ is
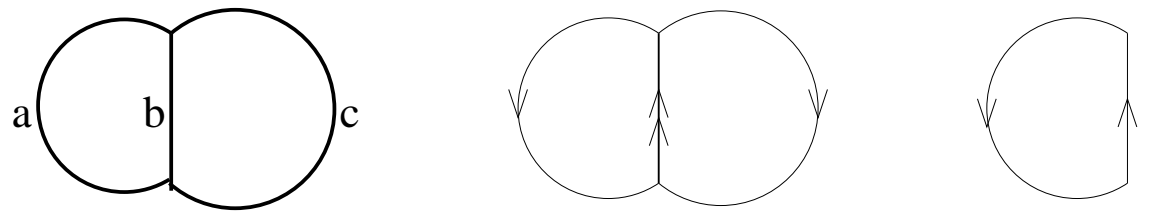

Figure 6. Two cycles $\gamma_{1}$ and $\gamma_{2}$ and their pairing $Q\left(\gamma_{1}, \gamma_{2}\right)=a+2 b$.

represented necessarily by the zero cycle, $Q$ descends to a symmetric bilinear from on $H_{1}(C, \mathbb{Z})$ (cf. Fig. [6), and consequently to a bilinear form on $\Omega(C)^{*}$. Clearly, $Q$ is non-degenerate and positive definite, and hence, provides an isomorphism $\tilde{Q}$ : $\Omega(C)^{*} \rightarrow \Omega(C)$. Under this isomorphism the lattice $\Lambda$ is mapped isomorphically to the integral forms $\Omega_{\mathbb{Z}}(C) \subset \Omega(C)$. Thus the form $Q$ makes $J(C)$ into a principally polarized tropical Abelian variety.

6.2. The Abel-Jacobi Theorem. Once and for all let us fix a reference point $p_{0} \in C$. Given a divisor $D=\sum a_{i} p_{i}$ we choose paths from $p_{0}$ to $p_{i}$. Integration along these paths defines a linear functional on $\Omega(C)$ :

$$
\hat{\mu}(D)(\omega)=\sum a_{i} \int_{p_{0}}^{p_{i}} \omega .
$$

For another choice of paths the value of $\hat{\mu}(D)$ will differ by an element in $\Lambda$. Thus, we get a well-defined tropical analog of the Abel-Jacobi map $\mu: \operatorname{Div}^{d}(C) \rightarrow J(C)$. Note that the Abel-Jacobi map $\mu$ does not depend on the choice of a base point $p_{0}$ 

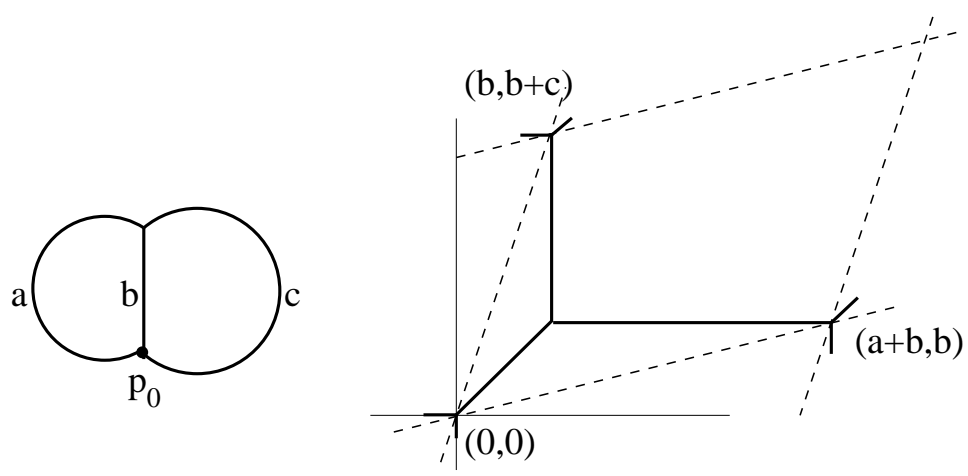

Figure 7. $\mu(C)$ in the tropical Jacobian $J(C)$.

if the degree $d$ of our divisor is zero. The dependence on $p_{0}$ for $d \neq 0$ will reappear later in solution to the Jacobi inversion.

Theorem 6.1 (Tropical Abel-Jacobi). For each degree $d$ the Abel's map $\mu$ factors through $\operatorname{Pic}^{d}(C)$ :

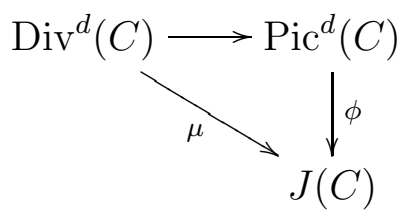

and $\phi$ is a bijection.

Proof. First we prove Abel's part: $\mu$ factors through and $\phi$ is injective. Theorem 6.4 below gives an explicit solution to the Jacobi inversion.

Let $D=\sum p_{i}-\sum q_{i}$ be a divisor of a rational function $f$. The gradient field $\nabla f$ defines a linear functional on $\Omega(C)$ via integration along its integral trajectories $t_{j}$ (counted with multiplicities). For some choice of paths from $p_{i}$ to $q_{i}$ this functional coincides with $\hat{\mu}(D)$. On the other hand, for any $\omega \in \Omega(C)$ we have

$$
\sum_{j} \int_{t_{i}} \omega=\int_{\tilde{Q}^{-1}(\omega)} d f=0 .
$$

Thus, $\mu(D)=0$ in $J(C)$.

Conversely, given any $D=\sum p_{i}-\sum q_{i} \in \operatorname{Div}^{0}(C)$ with $\mu(D)=0$ we can choose $k$ paths $\ell_{i}$ on $C$, such that each $\ell_{i}$ originates at $p_{i}$ and ends at $q_{i}$ and for any $\omega \in \Omega(C)$ we have $\sum_{i} \int_{\ell_{i}} \omega=0$. Now we fix $p_{0} \in C$ and define a rational function $f(x)$ on $C$ by choosing a path $\ell(x)$ from $p_{0}$ to $x$ :

$$
f(x):=\sum Q\left(\ell_{i}, \ell(x)\right) .
$$

Since $\sum Q\left(\ell_{i}, \gamma\right)=\sum \int_{\ell_{i}} \tilde{Q}(\gamma)=0$ for any closed loop $\gamma$ in $C$ the function $f(x)$ is independent of the choice of $\ell$. By construction $(f)=\sum p_{i}-\sum q_{i}$. 
6.3. Jacobi inversion. Given a tropical map $\phi: C \rightarrow X$ we can pull back any meromorphic function on $X$ to a meromorphic function on $C$, see e.g. [Mik06]. Now let $L$ be a line bundle and let $f$ be its section. Pulling back local representatives of $f$ defines a divisor $D$ on $C$ corresponding to some line bundle on $C$. Another section of $L$ defines a linearly equivalent divisor. Thus we can define a pull back line bundle $\phi^{*} L$ on $C$. Alternatively, to define $\phi^{*} L$ one can pull back a defining Čech cocycle of $L$.

Lemma 6.2. The Abel-Jacobi map $\mu: C \rightarrow J(C)$ is tropical.

Proof. We need to show that for any point $p \in C$ there is a local chart $U \subset R^{k-1}$ such that $\mu$ is the restriction of a $\mathbb{Z}$-affine map $\mathbb{R}^{k-1} \rightarrow \mathbb{R}^{g}$. But this is clear because affine linear coordinates near a $k$-valent vertex will also provide local coordinates in $\Omega(C)^{*}$ modulo some linear relations among cycles.

Next we will prove a refined version of the residue formula for curves in $\mathbb{R}^{g}$.

Lemma 6.3. Let $U$ be a connected open set of a curve tropically embedded in $\mathbb{R}^{N}$ with boundary $\partial U=\left\{z_{1}, \ldots, z_{k}\right\}$ and $\nu_{1}, \ldots, \nu_{k}$ - the corresponding outward primitive tangent vectors. Let $f$ be a rational function on $U$ with divisor $D=\sum a_{j} p_{j}$. Then

$$
\sum a_{j} p_{j}=\sum_{i=1}^{k}\left(\frac{\partial f}{\partial \nu_{i}}\left(z_{i}\right) \cdot z_{i}-f\left(z_{i}\right) \cdot \nu_{i}\right),
$$

where the summation and equality takes place in the vector space $\mathbb{R}^{N}$ and $p_{j}$ and $z_{i}$ are viewed as elements there.

Proof. The formula is additive with respect to gluing pieces and holds for affine functions. On the other hand, at $z=p_{i}$ the statement is the definition of $a_{i}$.

To state the tropical analog of the Jacobi inversion we denote by $\Theta_{\lambda}(x):=\Theta(x-\lambda)$ the translated theta function and let $\left[\Theta_{\lambda}\right]=[\Theta]+\lambda$ be its divisor. Denote by $L_{\lambda}$ the corresponding line bundle on $J(C)$ (it defines the same polarization as $L$ ). Both $\left[\Theta_{\lambda}\right]$ and $L_{\lambda}$ are well defined for a $\lambda \in J(C)$.

Theorem 6.4 (Jacobi Inversion). The pull back line bundle $\mu^{*} L_{\lambda}$ on $C$ has degree g. Moreover, if $D_{\lambda} \in \operatorname{Div}^{g}(C)$ denotes the pull back of the theta divisor $\left[\Theta_{\lambda}\right]$ to the curve, then there exists a universal $\kappa \in J(C)$ such that $\mu\left(D_{\lambda}\right)+\kappa=\lambda$ for all $\lambda \in J(C)$.

Proof. We use the fundamental domain $T$ of the universal covering of $C$. Specifying break points $z_{i} \in C$ determines $T$, and consequently, the lifting $\hat{\mu}: T \rightarrow \mathbb{R}^{g}$ of the Abel-Jacobi map $\mu$.

We consider the image of the fundamental domain $\hat{\mu}(\bar{T})$ in $\mathbb{R}^{g}$. A path connecting a pair $z_{i}^{-}, z_{i}^{+}$determines a cycle $\gamma_{i} \in \Lambda$, that is $\hat{\mu}\left(z_{i}^{+}\right)=\hat{\mu}\left(z_{i}^{-}\right)+\hat{\gamma}_{i}$ in $\mathbb{R}^{g}$. Using the 
functional equation we see that

$$
d \Theta_{\lambda}\left(\hat{\mu}\left(z_{i}^{+}\right)\right)-d \Theta_{\lambda}\left(\hat{\mu}\left(z_{i}^{-}\right)\right)=\tilde{Q}\left(\gamma_{i}\right) \in \Omega_{\mathbb{Z}}(C) .
$$

Set $\omega_{i}:=\tilde{Q}\left(\gamma_{i}\right)$ and note that $\left\langle\omega_{i}, \nu_{j}\right\rangle=\delta_{i j}$. Using the residue formula (5) we conclude that the total degree of $\mu^{*} L_{\lambda}$ is $\sum_{i=1}^{g} 1=g$.

The second statement follows from the refined residue formula (10) applied to the theta function restricted to $\hat{\mu}(T) \subset \mathbb{R}^{g}$ :

$$
\begin{aligned}
\hat{\mu}\left(D_{\lambda}\right)=\sum_{i=1}^{g}\left(\frac{\partial \Theta_{\lambda}}{\partial \nu_{i}}\left(\hat{\mu}\left(z_{i}^{+}\right)\right) \cdot \hat{\mu}\left(z_{i}^{+}\right)-\right. & \left.\Theta_{\lambda}\left(\hat{\mu}\left(z_{i}^{+}\right)\right) \nu_{i}\right) \\
& -\left(\frac{\partial \Theta_{\lambda}}{\partial \nu_{i}}\left(\hat{\mu}\left(z_{i}^{-}\right)\right) \cdot \hat{\mu}\left(z_{i}^{-}\right)-\Theta_{\lambda}\left(\hat{\mu}\left(z_{i}^{-}\right)\right) \nu_{i}\right) .
\end{aligned}
$$

Differentiating the right hand side with respect to $\lambda$ gives

$$
\sum_{i=1}^{g}\left(d \Theta_{\lambda}\left(\hat{\mu}\left(z_{i}^{+}\right)\right) \otimes \nu_{i}-d \Theta_{\lambda}\left(\hat{\mu}\left(z_{i}^{-}\right)\right) \otimes \nu_{i}\right)=\sum_{i=1}^{g} \omega_{i} \otimes \nu_{i}
$$

which is the identity element in $\operatorname{End}\left(\mathbb{R}^{g}\right)$. Thus, passing to the quotient $J(C)$ we get $\mu\left(D_{\lambda}\right)=\lambda+$ const.

6.4. Schottky problem and Torelli theorem. The space of all principally polarized tropical Abelian varieties is the same as the space of symmetric positive definite matrices modulo some discrete automorphism group action. So its dimension is $\frac{g(g+1)}{2}$. On the other hand the space of tropical curves of genus $g \geq 2$ is $3 g-3$. Hence the Jacobians form a subset $\mathcal{J}$ of positive codimension for $g \geq 3$ inside the space of all Abelian varieties. The Schottky problem is to describe this subset.

The naïve statement of the classical Torelli theorem fails for tropical curves. For instance, in Fig. 8 the polarized Jacobian does not see the length of the connecting edge in the genus 2 curve. However we conjecture that following reformulation holds:
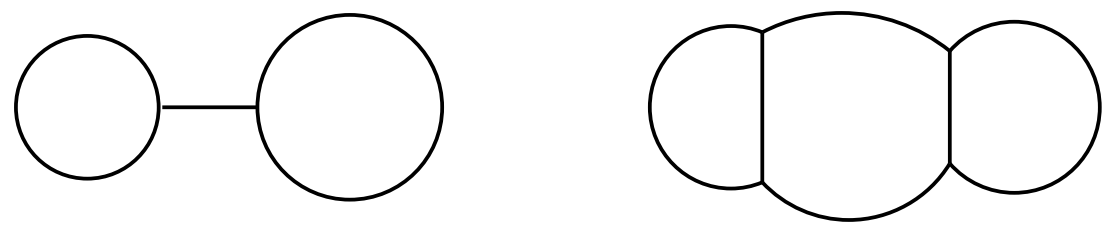

Figure 8. Counterexamples to Torelli in genus 2 and 3.

the map from the moduli space of tropical curves to $\mathcal{J}$ is tropical of degree 1. 


\section{Riemann-Roch Theorem}

In this section we study linear systems on a tropical curve $C$ of genus $g$ and prove tropical analog of the Riemann-Roch theorem.

Definition 7.1 (cf. BN06]). Let $D$ be an effective divisor on $C$. The dimension $\operatorname{dim}|D|$ of the linear system $|D|$ is the maximal integer $r \geq 0$ with the following property: for any effective divisor $R$ of degree $r$ the difference $D-R$ is effective. We let $\operatorname{dim}|D|=-1$ for non-effective $D$. We will equally use the cohomological notation $h^{0}(D)=\operatorname{dim}|D|+1$.

The definition immediately implies that for effective $D$ and $D^{\prime}$ one has

$$
h^{0}\left(D+D^{\prime}\right) \leq h^{0}(D)+\operatorname{deg} D^{\prime},
$$

and in particular, $h^{0}(D) \leq d+1$. By Jacobi inversion every divisor of degree $d \geq g$ is effective, which gives also the lower bound (vacuous for $d \leq g-1$ ):

$$
h^{0}(D) \geq d-g+1 .
$$

If the above becomes equality $D$ is called regular. A generic linear system with $d \geq g-1$ has to be regular since the preimage of a generic point $\mu(D) \in J(C)$ under the Abel-Jacobi map is of codimension $g$ in $\operatorname{Sym}^{d}(C)$. The discrepancy number

$$
\rho(D):=h^{0}(D)-d+g-1
$$

measures the lack of linearly independent relations among the points in $D$. An effective $D$ with $\rho(D)>0$ is called special.

Lemma 7.2. Every divisor $D$ of degree $d \geq 2 g-1$ is regular, i.e. $h^{0}(D)=d-g+1$.

Proof. Suppose $\operatorname{dim}|D|=s \geq d-g+1$. Consider a non-effective divisor $D^{\prime}$ of degree $d-s \leq g-1$ (it exists since the Abel-Jacobi map is not surjective on $\mathrm{Sym}^{k} C$ for $k \leq g-1)$. It can be written as $D^{\prime}=D-\left(D-D^{\prime}\right)=D-S$, where $S$ is of degree $s \geq g$ and hence effective. Consequently, $D^{\prime}$ must be effective which is a contradiction.

There seems to be no natural definition of the first cohomology $H^{1}(D)$. Thus to state the general Riemann-Roch theorem we adopt the Serre duality and introduce an analogue of the canonical divisor

$$
K:=\sum_{p \in C}(v(p)-2) p,
$$

where $v(p)$ is the valence of $p$. This is an effective divisor of degree $2 g-2$.

Remark. In the degeneration picture a $k$-valent vertex $p$ of $C$ corresponds to the Riemann sphere with $k$ punctures. This explains why the canonical divisor is defined as above: the number of zeros (the multiplicity of $p$ in $K$ ) of a rational form on $\mathbb{P}^{1}$ is the number of poles (valence of $p$ ) minus 2 . 
Theorem 7.3 (Riemann-Roch Theorem). $h^{0}(D)-h^{0}(K-D)=d-g+1$.

The strategy will be to proof the theorem for effective divisors of degree $g-1$ and then to use formal arguments in the Jacobian to deduce the general case. The goal of several lemmas below is to produce a particular form for a degree $g-1$ divisor. All graphs below are considered as subsets of a tropical curve $C$, that is, they are endowed with a $\mathbb{Z}$-affine structure.

Definition 7.4. Let $\Gamma$ be a (not necessarily connected) graph of relative genus $h$ (= the dimension of the space $H_{1}(\Gamma, \partial \Gamma)$ ). A framing for a point $p \in \Gamma$ is a choice of an edge which contains it. An effective divisor $D=p_{1}+\cdots+p_{k}$ with a framing for every point $p_{i}$ is called framed. A framed divisor $D=p_{1}+\cdots+p_{h}$ on $\Gamma$ is a moderator if every relative cycle $\gamma \in H_{1}(\Gamma, \partial \Gamma)$ contains some point $p_{i} \in D$ together with its framing.

The use of framing here is a convenient way to prevent a vertex of $\Gamma$ to be included in several cycles passing through it. In fact, the definition is equivalent to that for any choice of a basis of relative cycles $\gamma_{1}, \ldots, \gamma_{h}$ there is a reordering of the points such that $p_{i} \in \gamma_{i}$ for each $i$.

Lemma 7.5. Let $\Gamma$ be a graph with a moderator $D$ on it. Suppose that the restriction $D^{\prime}$ of $D$ to a connected open subset $\Gamma^{\prime} \subset \Gamma$ is a moderator on $\Gamma^{\prime}$. If $D^{\prime \prime}$ is another moderator on $\Gamma^{\prime}$, then $D-D^{\prime}+D^{\prime \prime}$ is a moderator on $\Gamma$.

Proof. Let $\gamma$ be a relative cycle on $\Gamma$. Then the restriction of $\gamma$ to $\Gamma^{\prime}$ is a relative cycle or empty. In the first case $\gamma$ contains a point of $D^{\prime \prime}$ and in the second it contains a point of $D-D^{\prime}$.

Definition 7.6. Let $\Gamma$ be a connected graph of relative genus $h$ with $\partial \Gamma \neq \emptyset$. An effective divisor $D=p_{1}+\cdots+p_{h+1}$ on $\Gamma$ is a terminator if its restriction $D_{0}$ to the closed core $\Gamma_{0}$ of $\Gamma$ (the maximal subgraph of $\Gamma$ with zero boundary) is a moderator on $\Gamma_{0}$, and $D-D_{0}=\partial \Gamma$.

It is clear from the definition that if $D$ is a terminator and $q \in \partial \Gamma$ then $D-q$ is a moderator on $\Gamma$. In fact Lemma 7.7 below shows that more is true. It reveals the true meaning of the term "terminator". For its proof it will be convenient to introduce a name for certain subsets of $\Gamma$.

Let $\Gamma$ be a connected graph, let $E$ be a connected subset of $\Gamma$, and $D-$ an effective divisor on $\Gamma$. Let $S$ be the subset of subdivisors $D^{\prime} \subset D$ with the following property: $E$ is contained in the closure of a connected component $\Gamma_{E}\left(D^{\prime}\right)$ of $\Gamma \backslash D^{\prime}$ whose boundary $\partial \Gamma_{E}\left(D^{\prime}\right)$ is contained in $\partial \Gamma \cup D^{\prime}$. We denote by $\Gamma(D, E) \subset \Gamma$ a minimal element among the components $\Gamma_{E}\left(D^{\prime}\right), D^{\prime} \in S$. (The choice of $\Gamma(D, E)$ is unique unless $E \subset D$.)

Lemma 7.7. Let $\Gamma$ be a connected graph and let $D$ be a terminator on it. Then for any $p_{0} \in \Gamma$ the divisor $D-p_{0}$ is linearly equivalent (relative the boundary) to a moderator. 
Proof. We use induction on the sum of the relative and absolute genera $h(\Gamma)+g(\Gamma)$. The statement is obvious for $h(\Gamma)=1$ and $g(\Gamma)=0$, that is when $\Gamma$ is an interval. By replacing $\Gamma$ with $\Gamma\left(D, p_{0}\right)$ we may assume that $\Gamma=\Gamma\left(D, p_{0}\right)$. For the induction step consider the meromorphic function on $\Gamma$

$$
f_{\epsilon}(x)=\min \{\epsilon, d(x, \partial \Gamma)\}
$$

where $\epsilon$ is the length of the shortest boundary edge(s) of $\Gamma$. If $v_{1}, \ldots, v_{k}$ are the interior ends of the shortest boundary edges, then $f_{\epsilon}$ provides linear equivalence (relative the boundary)

$$
D \sim D^{\prime}=D-\partial \Gamma+\partial \Gamma_{\epsilon}+v_{1}+\cdots+v_{k},
$$

where $\Gamma_{\epsilon}$ is the $\epsilon$-retraction of $\Gamma$. The induction step is to consider $\Gamma_{\epsilon}\left(D^{\prime}, p_{0}\right) \subset \Gamma_{\epsilon}$ with the terminator $D^{\prime \prime}=\left.D^{\prime}\right|_{\Gamma_{\epsilon}\left(D^{\prime}, p_{0}\right)}$ on it.

Any vertex $v_{i}$ which belongs to $\Gamma_{0}$ reduces the absolute genus, and any vertex that is not in $\Gamma_{0}$ reduces the relative genus. In any case $h\left(\Gamma_{\epsilon}\left(D^{\prime}, p_{0}\right)\right)+g\left(\Gamma_{\epsilon}\left(D^{\prime}, p_{0}\right)\right)<$ $h(\Gamma)+g(\Gamma)$. Hence, by the induction hypothesis $D^{\prime \prime}-p_{0}$ is equivalent to a moderator on $\Gamma_{\epsilon}\left(D^{\prime}, p_{0}\right)$.

Let $v_{1} \in \partial \Gamma_{\epsilon}\left(D^{\prime}, p_{0}\right)$ (there is at least one such vertex among the $v_{i}$ 's) and let $w_{1} \in$ $\partial \Gamma$ be the other end of this shortest edge. Next note that $D-w_{1}$ is a moderator on $\Gamma$, and consequently, so is $D^{\prime}-v_{1}$. On the other hand both $D^{\prime \prime}-v_{1}=\left.\left(D^{\prime}-v_{1}\right)\right|_{\Gamma_{\epsilon}\left(D^{\prime}, p_{0}\right)}$ and $D^{\prime \prime}-p_{0}=\left.\left(D^{\prime}-p_{0}\right)\right|_{\Gamma_{\epsilon}\left(D^{\prime}, p_{0}\right)}$ are equivalent to moderators on $\Gamma_{\epsilon}\left(D^{\prime}, p_{0}\right) \subset \Gamma$. Therefore by Lemma 7.5, the divisor $D^{\prime}-p_{0}$ is a moderator on $\Gamma$, and consequently, so is $D-p_{0}$.

Lemma 7.8. Let $\Gamma$ be a connected graph with $\partial \Gamma \neq \emptyset$, and $D-$ a moderator on it. Then given any point $p_{0} \in \Gamma$ there is a $q \in \partial \Gamma$ such that $D+q-p_{0}$ is linearly equivalent (relative the boundary) to a moderator on $\Gamma$.

Proof. Let $D^{\prime}$ be the restriction of $D$ to $\Gamma\left(D, p_{0}\right) \subset \Gamma$. Then $D^{\prime}$ is a moderator on $\Gamma\left(D, p_{0}\right)$, and consequently there is a unique $q \in \partial \Gamma\left(D, p_{0}\right)$ which is not in $D^{\prime}$, and hence $q \in \partial \Gamma$. Note that $D^{\prime}+q$ is a terminator on $\Gamma\left(D, p_{0}\right)$, and hence by Lemma 7.7. $D^{\prime}+q-p_{0}$ is equivalent to a moderator on $\Gamma\left(D, p_{0}\right)$. Finally, by Lemma 7.5, $D+q-p_{0}$ is a moderator on $\Gamma$.

The above lemma explains the essence of the term "moderator": given a negative point on $\Gamma$ the moderator can be used to "push" that negative point to the boundary of $\Gamma$.

Lemma 7.9. Let $D$ be an effective divisor on $C$ of degree $d \geq g-1$. Then there is a sequence of graphs $\emptyset=\Gamma_{0} \subset \Gamma_{1} \subset \cdots \subset \Gamma_{g-1} \subset \Gamma_{g}=C$ and a sequence of effective divisors $0=D_{0} \subset D_{1} \subset \cdots \subset D_{g-1}$ such that

(1) $\Gamma_{j}$ is a of relative genus $j$ and $D_{j}$ is an effective divisor on $\Gamma_{j}$ of degree $j$.

(2) $D-D_{j}$ is linearly equivalent to an effective divisor supported on the (possibly disconnected) curve $C_{j}=C \backslash \Gamma_{j}$. 
(3) $\bar{D}_{j}=\left(K-K_{C_{j}}\right)-D_{j}$ is equivalent (relative $\left.\partial \Gamma_{j}\right)$ to a moderator on $\Gamma_{j}$.

Proof. We use induction on $j$. All statements are trivial for $j=0$. Suppose we have $\Gamma_{j}$ and a moderator $\bar{D}_{j}$ on it. Consider an edge $E_{j+1}$ of $C_{j}$ containing a point $p_{j+1} \in D-D_{j}$. There are three possibilities (see Fig. 9):

- $\partial E_{j+1}=p+q \in K_{C_{j}}$ are two (possibly the same) points. Then we set $\Gamma_{j+1}=\Gamma_{j} \cup E_{j+1}$.

- $\partial E_{j+1}=p$. Then there is an edge $E_{j+1}^{\prime} \in C_{j}$ emanating from $p$ and we set $\Gamma_{j+1}=\Gamma_{j} \cup E_{j+1} \cup E_{j+1}^{\prime}$.

- $\partial E_{j+1}=\emptyset$. Then $E_{j+1}$ is a genus 1 component of $C_{j}$ and we set $\Gamma_{j}^{\prime}=$ $\Gamma_{j} \cup E_{j+1}$.
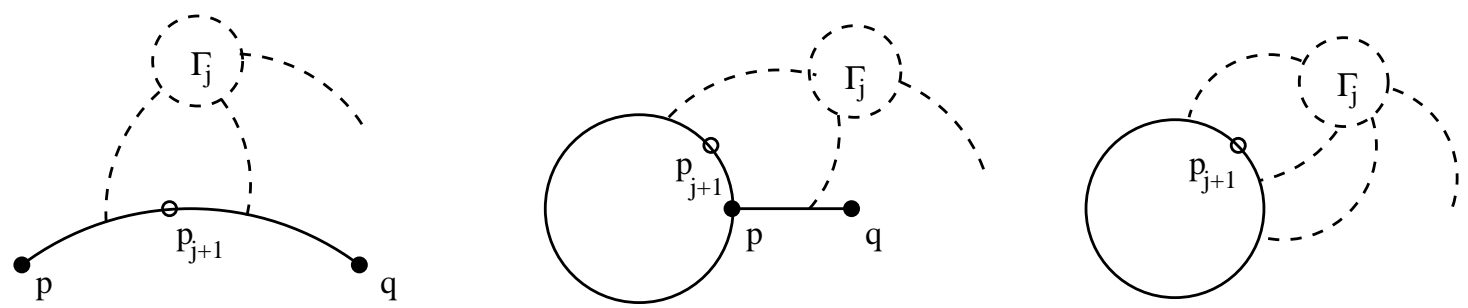

Figure 9. The three possibilities for $p_{j+1} \in E_{j+1}$.

We claim that in the last case one can replace $\bar{D}_{j}$ by a linear equivalent divisor which is a moderator on $\Gamma_{j}^{\prime}$. Then via Lemma 7.8 one can push $p_{j+1}$ out to the boundary of $\Gamma_{j}^{\prime}$ and repeat the step. After at most $g-1$ iterations we will be in one of the first two cases.

To prove the claim we consider $\Gamma_{j}\left(D, E_{j+1}\right)$ and use induction on $h\left(\Gamma_{j}\left(D, E_{j+1}\right)\right)+$ $g\left(\Gamma_{j}\left(D, E_{j+1}\right)\right)$ analogous to that of Lemma 7.7. The only difference is the first step in the induction: if $h\left(\Gamma_{j}\left(D, E_{j+1}\right)\right)=g\left(\Gamma_{j}\left(D, E_{j+1}\right)\right)=1$ then $\Gamma_{j}\left(D, E_{j+1}\right)$ is the loop with an edge attached, for which the claim is obvious.

For the first two cases we note that the divisor $\bar{D}_{j}+p$ is a moderator for the graph $\Gamma_{j+1}$, and $q \in \partial \Gamma_{j+1}$ is the compensating boundary point of Lemma 7.8 . Thus, $\bar{D}_{j+1}=\bar{D}_{j}+p+q-p_{j+1}$ is equivalent to a moderator on $\Gamma_{j+1}$. This finishes the induction step and the proof of lemma.

Proof of Riemann-Roch theorem. Because of Lemma 7.2 the Riemann-Roch holds for $d<0$ and $d>2 g-2$. Then by symmetry $D \leftrightarrow(K-D)$ it suffices to prove only the inequality

$$
h^{0}(K-D) \geq h^{0}(D)-d+g-1
$$

for $0 \leq d \leq 2 g-2$. The inequality is trivial for $\rho(D)=0$. Also applying (12) to $K-D$ shows that (14) holds for non-effective $D$. So from now on we assume that $D$ is special. 
We need to show that given any effective $R$ of degree $\rho(D)-1$ the divisor $K-D-R$ is effective. Replacing $D$ with $D+R$ reduces the statement to showing that $K-D$ is effective for any special $D$ of degree $d \geq g-1$.

The case $d=g-1$ follows immediately from the step $j=g-1$ of Lemma 7.9. Given Riemann-Roch for $d=g-1$ the statement for $d \geq g$ is then equivalent to the following claim. A divisor $D^{\prime}$ of degree $d^{\prime}<g-1$ is effective if for any effective $R$ of degree $r=g-1-d^{\prime}$ the divisor $D^{\prime}+R$ (of degree $g-1$ ) is effective. Indeed, then given a special $D$, the divisor $D-R$ is effective, and hence, so is $K-D+R$ by $\mathrm{RR}$ for $g-1$. Then the claim will imply that $K-D$ is effective as well.

We identify the set of degree $k$ effective divisors with the $k$-fold symmetric product Sym $^{k} C$ and let $W_{k}=\mu\left(\operatorname{Sym}^{k} C\right) \subset J(C)$, in particular $W_{1}=\mu(C)$. Then the above claim can be reformulated for a point $\lambda \in J(C)$ as $\lambda+W_{r} \subset W_{g-1} \Longrightarrow \lambda \in W_{g-1-r}$.

Let $F=\lambda+W_{r} \subset W_{g-1}$. For simplicity, assume that $F$ passes through a $(g-1)$ stratum $V$ of $W_{g-1}$ (if not, we apply the reasoning below to the corresponding smaller dimensional skeleton of $\left.W_{g-1}\right)$. Consider the subgraph $W_{V}$ of $W_{1}$ which is parallel to $V$ in $J(C)$. Each component of $W_{V}$ is a tree $T_{j}$. By the Euler characteristic count via projection $W_{1} \rightarrow S^{1}$ parallel to $V$ the number of these trees cannot be greater than $g-1$. Furthermore, this number is $g-1$ if we count each tree with the multiplicity equal to its relative genus.

Consider the case when each $T_{j}, j=1, \ldots, g-1$ is an interval. A point $x \in F \cap V$ then can be represented as $x=\lambda+z_{1}+\cdots+z_{r}$ where the $z_{i}$ are points from $r$ of these intervals. Once we fix the $r$ intervals there is at most one subset of the form $W_{r}+$ const contained in $W_{g-1}$ and passing through $x$ : this subset will be determined by the boundary of the stratum $V$ and the endpoints of the corresponding edges. On the other hand, through any $x \in W_{g-1}$ we have $\left(\begin{array}{c}g-1 \\ r\end{array}\right)$ subsets of type $z+W_{r}$ corresponding to choices of $r$ of the $g-1$ points on $C$ corresponding to $x$. Here $z$ is the sum of the remaining $g-1-r$ points in the decomposition of $x \in W_{g-1}$ and thus, $z \in W_{g-1-r}$. Since no non-trivial translation in $J(C)$ can leave $W_{r}$ invariant we must have $\lambda=z$.

If some of $T_{j}$ are trees of genus greater than 1 we consider continuous families of subsets of the form $W_{r}+$ const $\subset W_{g-1}$. However, the number of endpoints of these families is $g-1$ again and the rest of the proof is the same as in the interval case.

Remark. As we learned during the final revision of this manuscript, a different proof of this theorem was independently established by Gathmann and Kerber in GK06]. There they were able to deduce it from the combinatorial version of the RiemannRoch theorem due to Baker and Norine [BN06]. 


\section{Riemann's TheOREM}

In this section we will identify the subset $W_{g-1}=\mu\left(\operatorname{Sym}^{g-1} C\right) \subset J(C)$ with a translate of the theta divisor.

Theorem 8.1. $W_{g-1}+\kappa=[\Theta]$, where $\kappa \in J(C)$ is the constant from Theorem 6.4.

Proof. Since $\Theta(-x)=\Theta(x)$ the statement is equivalent to the following:

$\lambda \in[\Theta] \Longleftrightarrow$ there are $p_{1}, \ldots, p_{g-1} \in C$ such that $\lambda=-\kappa-\mu\left(p_{1}+\cdots+p_{g-1}\right)$.

$(\Longleftarrow)$ Pick any $p_{g} \in C$ and let $D=p_{1}+\cdots+p_{g}$. Then by the Jacobi inversion $\mu(p) \in[\Theta]+\kappa+\mu(D)$ if and only a point $p \in C$ is in the support of any $D^{\prime}$ in the linear system $|D|$. In particular, $\mu\left(p_{g}\right) \in[\Theta]+\kappa+\mu(D)$. Therefore

$$
-\kappa-\mu\left(p_{1}+\cdots+p_{g}\right)+\mu\left(p_{g}\right)=-\kappa-\mu\left(p_{1}+\cdots+p_{g-1}\right) \in[\Theta] .
$$

$(\Longrightarrow)$ By using the classical induction argument (cf., e.g., [Mum94, p.155]) we can see that $\mu(C) \not \subset\left[\Theta_{-\lambda}\right]$ is a generic condition for $\lambda \in[\Theta]$. That is to say that a generic linear system of degree $g$ is not special. So it is enough to show the statement for such a $\lambda$. By Jacobi inversion we can write it as $\lambda=-\kappa-\mu\left(p_{1}+\cdots+p_{g}\right)$. Consider $f(x)=\Theta(\lambda+\mu(x))$, a section of the line bundle $\mu^{*} L_{-\lambda}$ on $C$, whose divisor is precisely $D=p_{1}+\cdots+p_{g}$. On the other hand, $\lambda \in[\Theta]$, hence $p_{0}$ is in the support of the divisor of $f$. Therefore, some of the $p_{i}$ must coincide with $p_{0}$, and $\lambda$ has the desired form.
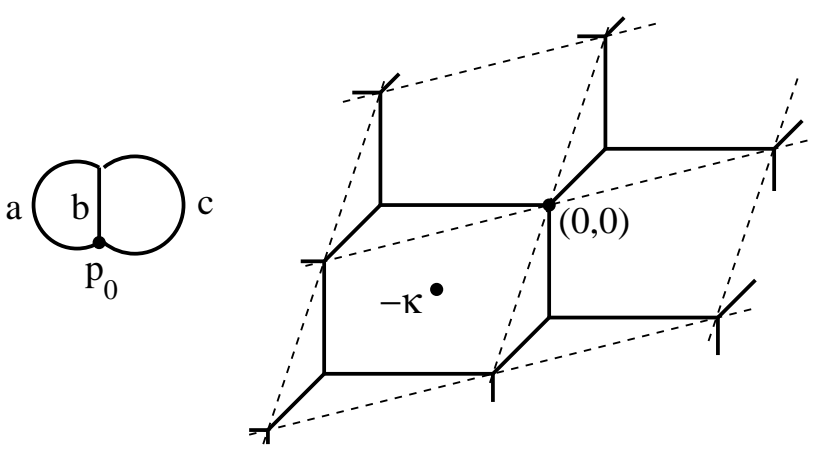

Figure 10. The theta divisor is the shift by $\kappa=\left(\frac{a+b}{2}, \frac{c+b}{2}\right)$ of the $W_{1}=\mu(C)$.

As a corollary we can identify $\kappa$. The Riemann-Roch implies that for any effective $D$ of degree $g-1$ the divisor $K-D$ is effective as well. Then

$$
W_{g-1}+\kappa=[\Theta]=-[\Theta]=-W_{g-1}-\kappa=W_{g-1}-\mu(K)-\kappa .
$$

But the theta line bundle defines a principal polarization, hence $[\Theta]$ cannot be stable under any non-trivial translation. Thus, $2 \kappa=-\mu(K)$. 


\section{REFERENCES}

[BHV01] Louis J. Billera, Susan P. Holmes, and Karen Vogtmann. Geometry of the space of phylogenetic trees. Adv. in Appl. Math., 27(4):733-767, 2001.

[BN06] Matthew Baker and Serguei Norine. Riemann-roch and abel-jacobi theory on a finite graph. http://arxiv.org/abs/math.CO/0608360, 2006.

[GK06] Andreas Gathmann and Michael Kerber. A riemann-roch theorem in tropical geometry. http://arxiv.org/abs/math.CO/0612129, 2006.

[GKZ94] I. M. Gel'fand, M. M. Kapranov, and A. V. Zelevinsky. Discriminants, resultants, and multidimensional determinants. Mathematics: Theory \& Applications. Birkhäuser Boston Inc., Boston, MA, 1994.

[GW00] Mark Gross and P. M. H. Wilson. Large complex structure limits of $K 3$ surfaces. J. Differential Geom., 55(3):475-546, 2000.

[Kap00] Mikhail Kapranov. Amoebas over non-archimedean fields. Preprint, 2000.

[KS01] Maxim Kontsevich and Yan Soibelman. Homological mirror symmetry and torus fibrations. In Symplectic geometry and mirror symmetry (Seoul, 2000), pages 203-263. World Sci. Publ., River Edge, NJ, 2001.

[Mik] Grigory Mikhalkin. Tropical geometry and amoebas. Book in preparation, http://www.math.toronto.edu/ mikha/TG-project.html.

[Mik05] Grigory Mikhalkin. Enumerative tropical algebraic geometry in $\mathbb{R}^{2}$. J. Amer. Math. Soc., 18(2):313-377 (electronic), 2005.

[Mik06] Grigory Mikhalkin. Tropical geometry and its application. Proceedings of the ICM 2006 Madrid, http://arxiv.org/abs/math.AG/0601041, 2006.

[Mum94] David Mumford. Tata lectures on Theta I. Birkhäuser Boston, 1994.

Mathematics Department, University of Toronto, Toronto, On M5S 2E4, Canada

E-mail address: mikha@math.toronto.edu

Mathematics Department, Harvard University, Cambridge, MA 02138, USA

E-mail address: zharkov@math.harvard.edu 